\title{
Saneamiento Ecológico: ¿Panacea o Caja de Sorpresas?
}

\author{
Miguel R. Peña V.*
}

* Ph.D., Profesor - Instituto CIMARA - Facultad de Ingeniería - Universidad del Valle, Santiago de Cali, Colombia.

E-mail:miguelpe@univalle.edu.co

Fecha de recepción: Abril 21 de 2004

Fecha de aprobación: Diciembre 17 de 2004

\section{RESUMEN}

El saneamiento ecológico (ECOSAM) se basa fundamentalmente en la separación del material semisólido (excreta) del líquido (orina). Trabajos previos argumentan que el proceso de deshidratación de la excreta parece eliminar de manera más efectiva los patógenos y por lo tanto los sistemas secos son mejores para reducir el riesgo microbiológico cuando se les compara con los métodos comunes para el manejo de excretas. Sin embargo, la literatura técnica reciente muestra que hace falta evidencia científica contundente que demuestre que la hipótesis ad hoc de eliminación de patógenos vía deshidratación es cierta en todas las condiciones ambientales posibles para la implementación de ECOSAM. Así mismo, la reducción del riesgo 
químico asociado con sustancias indeseables como los perturbadores endocrinos (PEs) que pueden estar presentes en la orina, es algo que se debe investigar a fondo en todas las tecnologías de saneamiento. Igualmente, la implementación de ECOSAM en un contexto social y cultural específico requiere de un trabajo comunitario importante para superar las limitaciones culturales que los usuarios potenciales podrían tener en relación con esta tecnología. Por lo tanto, ECOSAM no es una panacea para la solución de los problemas de contaminación y es básicamente una tecnología de saneamiento como cualquier otra que se encuentra en su etapa de desarrollo.

Palabras claves: Aguas residuales, excretas, saneamiento ecológico, sostenibilidad.

\section{ABSTRACT}

The concept of Ecological sanitation (ECOSAM) is based on the separation of materials flow. Thus, the solid (excreta) is separated from the liquid phase (urine). Previous work points out to the dehydration process as the key mechanism to eliminate pathogens effectively. In this sense, dry systems seem to be better to reduce microbiological risks when compared to more conventional methods for excreta management (i.e., sewerage, septic tanks, soaking pits etc). However, recent technical and scientific literature show there is a lack of strong evidence to demonstrate that the ad-hoc hypothesis of pathogens elimination via dehydration holds true in all possible environmental conditions where ECOSAM could be implemented. Furthermore, the reduction of chemical risks associated with undesirable substances such as the endocrine disruptors (EDs) which may be present in the urine is something that deserve in-depth scrutiny in all sanitation technologies. Additionally, the implementation of ECOSAM in a given social and cultural context requires a well-founded community work so as to overcome all the limitations that potential users may have in relation to this technology. Hence, ECOSAM is not the panacea for the solution of pollution problems and it is basically another sanitation technology at the development stage.

Key Words: Wastewater, excreta, ecological sanitation, sustainability.

\section{INTRODUCCIÓN}

La conceptualización del saneamiento ecológico (ECOSAM) surge de la necesidad de desarrollar métodos alternos a los comúnmente utilizados para el manejo y disposición de excretas y aguas residuales GTZ (2004, URL-1). La justificación para esto responde a razones tanto económicas como ambientales ya que en una sociedad cada vez más conciente del deterioro de los ecosistemas vitales, se vuelve importante la asignación y el uso racional de los recursos naturales, junto con los impactos provenientes de su explotación.

De otro lado, en Occidente se han utilizado esencialmente tecnologías de saneamiento con arrastre de agua tanto en sitio (i.e., tasa sanitaria con pozo de absorción e inodoro con tanque séptico) como en sistemas colectivos (i.e., alcantarillados convencionales) los cuales han sido primariamente concebidos sólo para transportar los desechos orgánicos fuera de la vivienda o del entorno público más inmediato sin importar necesariamente su tratamiento $y$ disposición final. En este sentido, ECOSAM es una tecnología que se concibe no sólo en función del tratamiento si no también para el reciclaje o recuperación de los nutrientes presentes en la orina y las excretas humanas. Esta diferencia en la concepción de la tecnología de saneamiento como un proceso de minimización, recuperación y reciclaje de desechos, enfoque de producción más limpia, más que un simple medio de reducir la contaminación al final del tubo, le define a estos materiales un valor económico intrínseco representado en su contenido de nutrientes (abono) y energía (biogás). Esrey et al. (2000) 
argumentan que ECOSAM es un sistema que ayuda a resolver algunos de los problemas más apremiantes de la sociedad como son la prevención de enfermedades infecciosas, la protección del ambiente y la recuperación y reciclaje de nutrientes y materia orgánica. En este sentido, por lo menos a nivel conceptual, ECOSAM presenta claramente ventajas importantes en relación con los otros métodos más convencionales para el manejo de excretas y aguas residuales.

Sin embargo, la experiencia particular en el sector de agua y saneamiento ( $A \& S$ ) a nivel mundial siempre ha demostrado que hay una gran distancia entre la elaboración teórica y la praxis en la realidad. Las dimensiones social y cultural son las que más afectan la aplicabilidad y en últimas la sostenibilidad de una solución tecnológica cualquiera en un contexto específico (Restrepo, 2002). De igual manera, condiciones ambientales particulares pueden hacer que una tecnología falle y esto no es razón suficiente para decir que la tecnología es mala per se. En contraposición con esta última afirmación, también existen argumentos que defienden a ultranza y de manera dogmática la aplicación indiscriminada de una tecnología cualquiera sin importar el contexto. Un ejemplo muy cercano de esta última afirmación la vivió nuestro país a mediados de la década de los 80s con la introducción de la tecnología anaerobia para el tratamiento de aguas residuales domésticas, la cual originó prácticamente una división de la ingeniería sanitaria en dos bandos: los aerobios y los anaerobios. Giraldo (1998) documenta muy bien esta división de credos en la práctica de la ingeniería de aguas residuales en nuestro país durante los 80 s y principios de los 90 s. Hoy día se acepta claramente, por ejemplo, que la tecnología anaerobia en un sistema integrado de tratamiento juega un papel importante en la recuperación de nutrientes, pero ella por si sola no es siempre la mejor opción.

Dado el auge que está tomando ECOSAM a nivel mundial pero también retomando nuestra propia historia y experiencia, el presente trabajo discute críticamente la potencialidad de esta tecnología en el Valle del Cauca centrándose en algunos aspectos claves de lo ambiental, lo tecnológico, lo social y lo cultural.

\section{MARCO TEÓRICO}

Ciclo del Agua y ECOSAM. La Figura 1 muestra una representación del ciclo antrópico del agua incluyendo la concepción de ECOSAM.

Producción más limpia (PML) y ECOSAM. Dos principios de la filosofía de PML están muy relacionados con ECOSAM, ellos son: la minimización de residuos en la fuente y la separación de los mismos para facilitar su manejo adecuado. En el primer caso, ECOSAM ha sido concebido fundamentalmente para trabajar sin agua o con una cantidad mínima de ella, además las soluciones de ECOSAM son descentralizadas y se construyen en el ámbito de la vivienda que es el núcleo primario de producción de desechos (Esrey et al., 2000). En relación con el segundo principio, la separación de la orina y las excretas permite un mejor manejo de estos flujos de materiales (nutrientes y materia orgánica) en función de su recuperación y uso último. Por lo tanto, ECOSAM es un método de manejo de desechos que incorpora principios de PML.

Gestión Integrada de Recurso Hidrico (GIRH) y ECOSAM. En términos de GIRH, ECOSAM presenta una interrelación directa entre el sector de $A \& 5$ y el sector Agrícola con particular énfasis en la producción y seguridad alimentaria en este último. Así mismo, varios autores defienden los impactos positivos de ECOSAM en la conservación de la calidad del agua tanto subterránea como superficial [SIDA, 1999; Esrey et al., 2000; GT2 (2004, URL-1)] y esto puede tener implicaciones positivas para la explotación comercial de otros usos del recurso como el turístico, la generación de energía eléctrica y diversos usos industriales. ECOSAM puede ser 
por lo tanto, una herramienta tecnológica que coadyuva a la gestión de la calidad del agua en un marco amplio de GIRH.

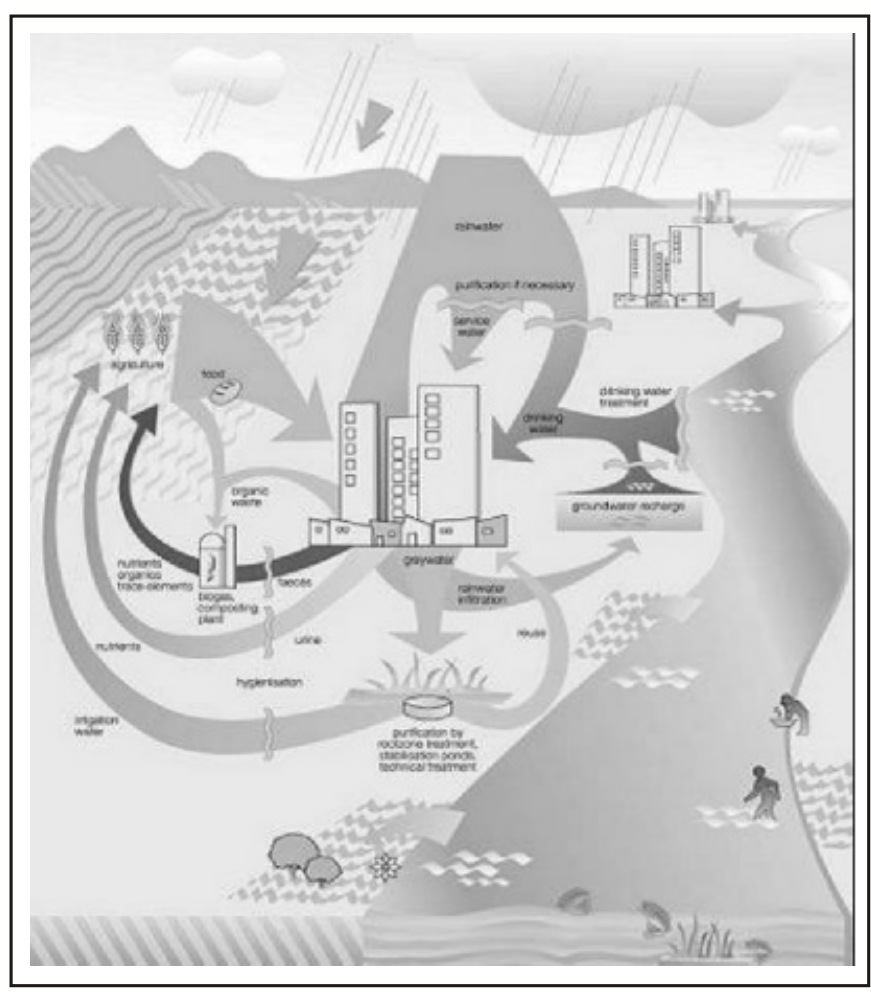

Figura 1. Ciclo antrópico del agua incluyendo ECOSAM. Fuente:GTZ (2004, URL-1)

ECOSAM se basa fundamentalmente en la separación del material semisólido (excreta) del líquido (orina). Una vez separados estos flujos, la excreta es sometida al proceso de deshidratación el cual depende de una adecuada temperatura, de las condiciones de ventilación, pero sobre todo de la humedad relativa en el ambiente. En algunos casos, para mejorar la deshidratación se adicionan materiales higroscópicos (absorbentes de humedad) como la cal en sus formas viva o hidratada $\left[\mathrm{CaO} \circ \mathrm{Ca}(\mathrm{OH})_{2}\right]$.

De otro lado, la orina básicamente se almacena en depósitos desde los cuales se procede a su distribución o uso final para riego de cultivos. En términos generales, las excretas son las que contienen los microorganismos patógenos de importancia en salud pública (i.e., viruses, bacterias, hongos, protozoarios y helmintos). Usualmente la orina no tiene patógenos pero sí contiene la mayor cantidad de los nutrientes reutilizables. A nivel del paquete tecnológico propiamente dicho, ECOSAM consta de unidades sanitarias de evacuación con dispositivos de separación de excretas y orina. La Figura 2 muestra fotografías de una unidad sanitaria típica con su respectivo dispositivo de evacuación desarrollados en el marco del Proyecto Piloto de Saneamiento Ecológico en Palestina (PPSEP).

En el documento publicado por SIDA (1999), Ios autores argumentan que el proceso de deshidratación parece eliminar de manera más efectiva los patógenos y por lo tanto los sistemas secos son mejores para reducir el riesgo microbiológico cuando se les compara con los métodos comunes para el manejo de excretas.

Mótese entonces que la aplicación de ECOSAM parece ser óptima en zonas de baja humedad relativa y altas temperaturas, lo que define climas áridos donde el agua es un recurso escaso. Así mismo, estas condiciones climáticas adversas limitan el desarrollo de la agricultura con lo cual se tienen usualmente asentamientos humanos muy pobres, con serios problemas de nutrición e higiene tanto a nivel personal como doméstico.

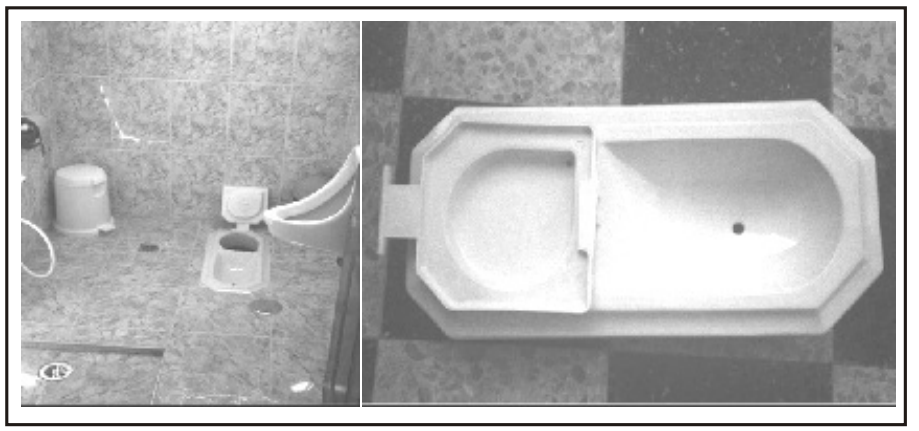

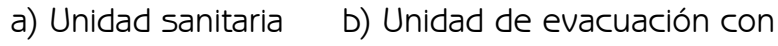
completa. separación de orina.

Figura 2.Fotografía de una unidad de evacuación para ECOSAM.

Fuente:Proyecto PPSEP (Wimblad, 2002). 


\section{VENTAJAS Y DESVENTAJAS DE ECOSAN}

La Tabla 1 resume las principales ventajas y desventajas de ECOSAM con base en referencias encontradas en la literatura técnica.

Tabla 1. Principales ventajas y desventajas de ECOSAM

\begin{tabular}{|c|c|}
\hline Ventajas & Desventajas \\
\hline Disminución de la contaminación ambiental $^{*}$ & $\begin{array}{l}\text { Restricciones de tipo ambiental para su } \\
\text { aplicación exitosa (i.e., humedad relativa alta, }\end{array}$ \\
\hline Reducción del consumo de agua * & $\begin{array}{l}\text { zonas de alta pluviosidad y niveles freáticos } \\
\text { altos) }\end{array}$ \\
\hline Reducción del uso de fertilizantes * & La deshidratación de las excretas no \\
\hline $\begin{array}{l}\text { Reuso directo de los nutrientes de la orina }(\mathrm{N}, \mathrm{P} \text {, } \\
\mathrm{K})\end{array}$ & $\begin{array}{l}\text { garantiza una eliminación segura de los } \\
\text { patógenos }\end{array}$ \\
\hline $\begin{array}{l}\text { Uso de las excretas como acondicionador del } \\
\text { suelo }\end{array}$ & $\begin{array}{l}\text { Eventualmente se requiere la adición de un } \\
\text { material deshidratante para lograr el buen }\end{array}$ \\
\hline $\begin{array}{l}\text { ECOSAN a gran escala podría contribuir a la } \\
\text { reducción del calentamiento global }{ }^{+}\end{array}$ & $\begin{array}{l}\text { funcionamiento de ECOSAN y esto } \\
\text { representa costos para el sistema }\end{array}$ \\
\hline No hay producción de olores ni moscas & \multirow{4}{*}{$\begin{array}{l}\text { ECOSAN requiere de una cultura del manejo } \\
\text { de los desechos humanos como un recurso } \\
\text { productivo y esta no necesariamente existe } \\
\text { en el imaginario de la cultura latinoamericana } \\
\text { a diferencia del lejano oriente }\end{array}$} \\
\hline $\begin{array}{l}\text { Los sistemas de ECOSAN no presentan peligro } \\
\text { para los niños por su baja profundidad }\end{array}$ & \\
\hline Bajos costos de construcción, O\&M & \\
\hline Incremento rápido de la cobertura sanitaria & \\
\hline
\end{tabular}

Fuente: (SIDA, 1999; Esreyet al., 2000).

Ventajas que son comunes a varios sistemas de saneamiento en sitio (i.e., letrinas mejoradas ventiladas del tipo levantadas o composteras de hoyo desplazado).

+ Ésta es una ventaja discutible ya que debería tenerse el balance de masa alrededor del valor nutricional total de la excreta humana y ver si dicha cifra es suficiente para mantener la productividad agrícola necesaria para sostener la población mundial y adicionalmente un excedente para recuperar las zonas deforestadas del planeta que serían las que en últimas contribuirían a la verdadera disminución del $\mathrm{CO}_{2}$ atmosférico vía fotosíntesis. Mótese que el aporte de los cultivos transitorios de explotación agrícola a la disminución del $\mathrm{CO}_{2}$ no puede equipararse a la de un ecosistema boscoso permanente.

\section{DISCUSIÓN}

Aspectos ambientales y tecnológicos. Como se dijo anteriormente, el correcto funcionamiento de ECOSAM depende de unas condiciones ambientales que permitan ante todo la deshidratación natural de la excreta depositada en el pozo de almacenamiento. Entre tanto, la orina almacenada en el reservorio debe diluirse con agua para su posterior reuso en irrigación de cultivos. Bajo estos preceptos, ya existen varios proyectos de ECOSAM en el mundo que reportan resultados prometedores [GT2 (2004, URL-1); Castillo, 2002]. Las experiencias mejor documentadas están en México, Palestina y Zimbabwe. Así mismo, en Latinoamérica ya se está introduciendo el concepto de ECOSAM en varios países de la Región (Rosensweig y Pérez, 2002).

Sin embargo, en relación con lo ambiental no existe en la literatura técnica disponible un ejemplo o evaluación de ECOSAM en aquellos sitios con humedades relativas altas o regímenes de pluviosidad elevada. Tal vez la única referencia a este respecto hace mención a la unidad sanitaria desarrollada por el Centro para el Desarrollo Limpio (CDL) el cual está destinado a los países insulares del Pacífico sur (SIDA, 1999). Mótese que este sistema en realidad no responde a los conceptos de ECOSAM y hace énfasis en la no descarga, más que en la separación y reciclaje de orina.

De otro lado, la deshidratación como el principal mecanismo de eliminación de patógenos en ECOSAM es algo que merece una mayor revisión, discusión y evaluación a nivel científico en los 
sistemas existentes. En este sentido, la deshidratación como tal produce un cese de la actividad biológica asociada con la excreta y su contenido de humedad, y eventualmente puede eliminar gran parte de la matriz microbiológica presente en este material. Sin embargo, algunas especies bacteriales patógenas como Clostridium perfringens y Clostridium botulinum (que pueden estar presentes en las excretas y entrar en contacto con los alimentos) desarrollan esporulación como mecanismo de protección ante condiciones ambientales adversas y entran en un periodo de latencia hasta que encuentran nuevamente condiciones propicias para su reactivación FDA (2004, URL-2). Del mismo modo, en un coloquio de la Academia Americana de Microbiología se presentó y discutió evidencia científica de las condiciones ambientales extremas bajo las cuales existe vida y actividad microbiana en el planeta. Por ejemplo, en habitats hostiles con $\mathrm{pH}>12$, ausencia de oxigeno y muy baja actividad hídrica (humedades relativas 55\%) se han encontrado eubacterias alcalifilicas, archaeas, hongos anaeróbicos, protozoarios y hongos xerofilícos que viven y desarrollan actividad metabólica (Staley et al., 1996).

Dentro de los habitats naturales que tienen estas características ambientales están los suelos desérticos, lagos alcalinos y salmueras. Por lo tanto, pareciera que la deshidratación no es una condición suficiente para la eliminación total de la vida microbiana, quedando aún por descubrir que patógenos potenciales pueden existir en estas condiciones donde hay organismos extremofilos viables. Ciertamente hace falta evidencia científica contundente que demuestre que la hipótesis ad hoc de eliminación de patógenos vía deshidratación es cierta en todas las condiciones ambientales posibles para la implementación de ECOSAM. Mótese que otros patógenos resistentes a condiciones ambientales adversas como los huevos de helmintos y algunos viruses pueden también sobrevivir a la deshidratación como único medio de eliminación.
Otro aspecto importante de riesgo para la salud que está tomando auge en relación con la contaminación producida por aguas residuales y excretas tiene que ver con los llamados perturbadores endocrinos (PE) o Endocrine Disruptors (ED) por su sigla en Ingles. Estos compuestos son químicos sintéticos o naturales que interfieren con el balance hormonal normalmente requerido para las funciones biológicas de los mamíferos incluida la especie humana (Jesperson, 2003). Los desbalances hormonales causados por estos compuestos químicos generan desordenes variados del sistema reproductivo tales como la feminización de los machos o la masculinización de las hembras, lo cual ha sido observado principalmente en peces [Cone (1998) citado por Esrey y Andersson (1999); Jesperson, 2003].

Raloff (1998) reporta la identificación de fármacos y sus metabolitos en varios sistemas de abastecimiento de agua de Europa y la única explicación plausible para su presencia en dichos sistemas, es que ellos provienen de los excrementos humanos. Este autor menciona la presencia de drogas reguladoras de lípidos como el fenazone, analgésicos como el ibuprofeno, varios quimioterapéuticos, antibióticos, hormonas, drogas para el corazón y hasta drogas siquiátricas para el control de la epilepsia. Es posible que entre el 50 al 90\% del fármaco ingerido sea excretado en forma biológicamente activa, mientras que la fracción parcialmente degradada puede ser activada nuevamente a través de reacciones químicas en el ambiente. Los riesgos de salud a mediano y largo plazo que provienen de estos contaminantes aún se desconocen tal como lo plantea Cone (1998). Sin embargo, muchos críticos señalan que no hay pruebas contundentes de que los PE afecten a los humanos de alguna manera. En contraposición, muchos científicos argumentan que no se debe esperar a la aparición de una generación de hermafroditas para exigirles a las autoridades ambientales que empiecen a investigar o continúen investigando sobre este riesgo tan 
crítico. Para una información mas detallada y actualizada sobre esta temática se recomienda consultar Jesperson (2003).

A nivel conceptual parece lógico que ECOSAM es una buena estrategia para reducir el riesgo microbiológico y nuevos riesgos como el producido por los PEs. La deshidratación parece ser por lo tanto un buen mecanismo físico para detener el flujo de patógenos, nutrientes y demás sustancias que coexisten en la matriz de composición de la excreta. Sin embargo, como ya se dijo anteriormente, la deshidratación detiene la actividad biológica y con ella las posibilidades de biodegradación de los diferentes contaminantes suspendidos y disueltos en la fase líquida (incluida muy seguramente una fracción de los PEs) de la excreta. Esto es perfectamente aceptable si luego de la deshidratación, todo este material disecado no se reutilizase como abono en cultivos. Pero es precisamente esta interacción con el ambiente la que nos lleva a plantearnos serios interrogantes como:

¿Qué sucede si este material seco, aparentemente inocuo, al entrar en contacto nuevamente con la humedad natural del suelo o del aire o por fenómenos de precipitación se rehidrata? Será posible que compuestos químicos indeseables como los PEs se resolubilicen en la humedad adquirida en estos procesos naturales de interacción y continúen su flujo por el ambiente? Qué pasa con el crecimiento y la actividad biológica que posiblemente se reactiven con las nuevas condiciones de humedad y el contacto con la tierra más aun sabiendo que un gramo de suelo contiene una diversidad microbiana inimaginable?

De otro lado, en la literatura citada con anterioridad no hay una referencia específica a la presencia de los PEs en la orina, cuando se sabe que ésta es una de las principales vías de expulsión de los metabolitos generados por el cuerpo humano (Arboleda, 2004; Madera, 2004). Mótese que el reuso directo de la orina en agricultura podría además de recuperar los nutrientes, potencializar el flujo de contaminantes indeseados como los PEs en el ambiente.

Pareciera entonces que con el nivel actual de conocimiento científico y tecnológico alrededor de los procesos claves de ECOSAM que son la separación de la orina y la deshidratación de la excreta, quedan aún muchas preguntas por contestar de manera satisfactoria y los invocados beneficios de esta tecnología (como son la recuperación y reuso de nutrientes y materia orgánica, junto con la eliminación de organismos patógenos) se ven seriamente cuestionados cuando se someten al escrutinio cuidadoso del método científico y al conocimiento vigente en otras ramas de las ciencias naturales como son la farmacología, la ecología microbiana y la bioremediación de contaminantes. Podría entonces suceder que en últimas los beneficios logrados dentro de los sistemas de ECOSAM se pierdan cuando los materiales reutilizables sean reintroducidos libremente en el ambiente y el resultado último en términos de riesgos para la salud y el ambiente sería equiparable al de otros sistemas de saneamiento.

De otro lado, en la poca literatura que existe sobre ECOSAM, se describe principalmente la parte técnica de los sistemas implementados y el proceso de construcción, arranque, operación y mantenimiento de los mismos. Sin embargo, hay una escasez de estudios esencialmente sobre la evaluación de las supuestas ventajas de esta tecnología. Un ejemplo específico de esto es la medición de los impactos en salud al remover efectivamente los patógenos por deshidratación, ya que este mecanismo aún no está suficientemente demostrado por datos $y$ permanece como una hipótesis ad hoc. De otro lado, la efectividad de ECOSAM para el control o reducción de los PEs, es algo aún menos documentado ya que este es un riesgo crónico para la salud que toma importancia para la ciencia y la tecnología del agua a partir de dos trabajos pioneros publicados por Tinnin (1997) y Mulroy 
(2000), los cuales fueron ganadores del premio Estocolmo del Agua en la categoría de Joven Investigador en sus respectivos años. Sólo a partir de ese momento se comienza un trabajo que hasta ahora está generando las primeras publicaciones internacionales que sientan las bases para un trabajo riguroso alrededor de esta temática novísima IWA (2004, URL-3; 2004, URL4). Las referencias actuales generalmente describen y documentan solamente casos y procesos de implementación de ECOSAM sin profundizar en los aspectos más científicos sobre los cuales este documento intenta reflexionar de manera crítica. Para aquellos lectores interesados en estas temáticas generales se recomienda consultar (SIDA, 1999; Esrey y Andersson, 1999; Castillo, 2002 y Wimblad, 2002).

Aspectos sociales y culturales. Un aspecto fundamental para la sostenibilidad de cualquier tecnología tiene que ver con el grado de apropiación de la misma, lo que a su vez está estrechamente ligado con la participación de la comunidad en todo el proceso de búsqueda y toma de decisiones alrededor de la solución de saneamiento requerida. En este sentido, la provisión de soluciones con base en la demanda y las preferencias de los usuarios son aspectos centrales para garantizar el éxito de cualquier tecnología de saneamiento (Restrepo, 2002).

Sin embargo, la satisfacción de la demanda con una solución particular de saneamiento teniendo en cuenta las preferencias de la gente en cuanto a comodidad, status social, costos razonables (de inversión, O\&M) y requerimientos de operación y mantenimiento, son variables que responden fundamentalmente a la cultura y el nivel socioeconómico de la población usuaria. En este sentido, ECOSAM parece cumplir con todos estos requisitos a excepción del último, esto es, los requerimientos de O\&M. En general, los sistemas de ECOSAM aunque son sencillos para operar y mantener requieren una mayor atención por parte de los usuarios para que el proceso de deshidratación de la excreta y el reuso del humus resultante y la orina se den adecuadamente. Esto último implica actividades de manipulación y contacto eventual con estos materiales, para lo cual es necesario que los usuarios acepten y desarrollen dichas prácticas de manera segura.

La manipulación de desechos tanto líquidos como sólidos es una actividad culturalmente rechazada en el contexto de la cultura Latinoamericana, y de alguna manera esto puede generar discriminaciones o estigmas sociales a quienes desarrollan dichas tareas en una comunidad pequeña. En contraste, la cultura oriental (i.e., China, Corea, Japón, Tailandia y Vietnam) ha incorporado a través de tradiciones milenarias el reuso y reciclaje de excrementos tanto humanos como animales y en dicho contexto tecnologías como ECOSAM pueden tener una mayor aceptación por parte de la gente. Esta situación lleva a que la implementación y el éxito final de ECOSAM en contextos sociales, económicos y ambientales tan variados como el Latinoamericano, requiera de un gran trabajo de sensibilización, participación y educación de la comunidad como estrategia para lograr la sostenibilidad de esta tecnología. Por supuesto, existirán siempre excepciones dado que en varios países de la región también coexisten regiones áridas y semiáridas con comunidades pobres y escasez de recursos naturales donde ECOSAM podría ser al igual la excepción más no la regla.

\section{CONCLUSIONES}

ECOSAM no es una panacea para la solución de los problemas de contaminación, es básicamente una tecnología de saneamiento como cualquier otra que se encuentra en su etapa de desarrollo y requiere de un mayor trabajo de investigación científica y tecnológica para resolver los interrogantes relacionados con el manejo del riesgo microbiológico y del riesgo químico por compuestos indeseables como los PEs.

Las publicaciones recientes sobre el riesgo potencial asociado a los PEs en el ambiente llevan 
a repensar los aspectos de valoración y reducción de los riesgos microbiológico y químico en relación con todas las tecnologías de saneamiento como barreras de control de la contaminación y ECOSAM no es la excepción.

Los aspectos culturales y sociales relacionados con la sostenibilidad de ECOSAM juegan un papel central en la aceptación y éxito de esta tecnología en el caso particular de Latinoamérica, dada nuestra diversidad cultural, social, étnica y ambiental.

Finalmente y a manera de reflexión pareciera que la verdadera solución a los problemas graves de contaminación que se auguran para esta sociedad postmoderna no está en el desarrollo de tecnologías cada vez más sofisticadas, por el contrario, pareciera que la verdadera solución está en que la especie humana redescubra su armonía con la naturaleza que una vez nos permitió erguirnos sobre nuestros pies y racionalizar el mundo para hacerlo mejor y no para destruirlo inexorablemente.

\section{REFERENCIAS}

■ Arboleda, C.E. (2004). Comunicación personal. MD Especialista en Biología y Endocrinología de la Reproducción. Santiago de Cali, Colombia.

■ Castillo, L. (2002). Sanitario Ecológico Seco: Manual de diseño, construcción, uso y mantenimiento. Guadalajara, México.

$\square$ Cone, M. (1998). River pollution study finds hormonal defects in fish. Los Angeles Times. September 22. Los Angeles, CA. USA.

$\square$ Esrey, A. Y Andersson, I. (1999). Saneamiento Ambiental desde una Perspectiva de Ecosistema. En: Ecological Sanitation: Closing the Loop for Food Security. Ahuatepec, México.

घ Esrey, A., Andersson, I., Hillers, A and Sawyer, R. (2000). Ecological Sanitation Closing the Loop to Food Security. Report from UMDP/SEED. Mew York, USA. $\square$ Esrey, A., Andersson, I., Hillers, A. and Sawyer, R. (2000). Ecological Sanitation Closing the Loop to Food Security. Report from UMDP/SEED. Mew York, USA.

■ Giraldo, E. (1998). Perspectivas del tratamiento anaerobio de las aguas residuales domesticas en Colombia. Conferencia Internacional Agua 1998. En: Memorias del Seminario Internacional en Saneamiento Básico y Sostenibilidad. Universidad del Valle, Instituto Cinara. Santiago de Cali, Colombia.

$\square$ Jesperson, K. (2003). Endocrine Disruptors. What are they doing to you?. On Tap: Drinking Water Mews for America's Small Communities. Mational Environmental Services Centre. West Virginia University. USA.

$\square$ Madera, J. (2004). Comunicación personal. MD Especialista en Gerencia Hospitalaria. Santiago de Cali, Colombia.

$\square$ Mulroy, A. (2000). On the inefficiency of wastewater treatment processes and its relation to antibiotic contamination and bacterial resistance in American waterways. Winner of the Stockholm Junior Water Prize. SIWI. Stockholm, Sweden.

$\square$ Raloff, J. (1998). Drugged waters: Does it matter that pharmaceuticals are turning up in water supplies? Science Mews 153:187-189.

$\square$ Restrepo, I. (2002). Team learning projects as a strategy to contribute to the sustainability of water supply and sanitation services. Ph.D thesis. School of Civil Engineering, University of Leeds. Leeds, UK.

$\square$ Rosensweig, F. and Pérez, E. (2002). Improving sanitation in small towns in Latin America and The Caribbean. Environmental Health Project (EHP). Strategic Report 3. USAID. Washington DC. USA.

$\square$ SIDA (1999). Saneamiento Ecológico. Agencia Sueca de Cooperación Internacional para el Desarrollo/Fundación Friedrich Ebert. Granada, México.

$\square$ Staley, J.T., Castenholz, R.W., Colwell, R.R., Holt, J.G., Kane, M.D., Pace, M.R., Salyers, 
A.A. and Tiedje, J.M. (1996). The Microbial World: Foundation of the Biosphere. A report from the American Academy of Microbiology. Palm Coast, FL. USA.

च Tinnin, S. (1997). On the correlation between the reproductive rate of sea urchins and water pollution. Winner of the Stockholm Junior Water Prize. SIWI. Stockholm, Sweden.

$\square$ Wimblad, U. (2002). Ecological Sanitation Pilot Project in Palestine- A project appraisal. SIDA consultant report. Hebron and Kyoto.

$\square$ URL 1. GT2 (2004).

http://www.gtz.de/ecosan/english/index.html . Ecological Sanitation: Closing the loop in wastewater management and sanitation. Visitado 08/03/2004.

$\square$ URL 2. FDA (2004).

http://vm.cfsan.fda.gov/ mow/chap11.html . Foodborne pathogenic microorganisms and natural toxins handbook. Visitado 09/03/2004.

$\square$ URL 3. IWA (2004). http://www.iwaponline.com/wio/2004/05/wio 200405af90940f.html. Assessment of Waters for Estrogenic Activity. Visitado 10/04/2004.

$\square$ URL 4. IWA (2004).

http://www.iwaponline.com/wio/2003/01/wio 200301wf99eco3.html. Evaluating Endocrine Disruption in Receiving Waters: Screening for Biomarkers. Visitado 10/04/2004. 